\title{
Accurate and efficient method for the treatment of exchange in a plane-wave basis
}

\author{
A. Sorouri, ${ }^{a)}$ W. M. C. Foulkes, and N. D. M. Hine \\ Blackett Laboratory, Imperial College, Prince Consort Road, London SW7 2AZ, United Kingdom
}

(Received 19 October 2005; accepted 16 December 2005; published online 9 February 2006)

\begin{abstract}
We describe an accurate and efficient extension of Chawla and Voth's [J. Chem. Phys. 108, 4697 (1998)] plane-wave based algorithm for calculating exchange energies, exchange energy densities, and exchange energy gradients with respect to wave-function parameters in systems of electrons subject to periodic boundary conditions. The theory and numerical results show that the computational effort scales almost linearly with the number of plane waves and quadratically with the number of $k$ vectors. To obtain high accuracy with relatively few $k$ vectors, we use an adaptation of Gygi and Baldereschi's [Phys. Rev. B 34, 4405 (1986)] method for reducing Brillouin-zone integration errors. (C) 2006 American Institute of Physics. [DOI: 10.1063/1.2166016]
\end{abstract}

\section{INTRODUCTION}

Most of today's computational studies of the quantum mechanics of electrons in large molecules and solids are based on Hohenberg-Kohn-Sham density-functional theory (DFT). ${ }^{1-4}$ This shows that the ground-state energy of a many-electron system may be obtained by minimizing a functional $E[n]$ of the electron density $n(\mathbf{r})$. The energy functional is normally divided into three parts: a noninteracting kinetic energy, a Coulomb energy due to the classical electrostatic interactions of the nuclear and electronic charge densities, and a term called the exchange-correlation energy that contains all other many-body interactions. The electrostatic contribution may be calculated directly from the density and the kinetic energy obtained by solving a noninteracting Schrödinger equation, but the exchange-correlation functional $E_{\mathrm{xc}}[n]$ is not known exactly and must be approximated. The simplest approximations, the local-density approximation $^{2}$ (LDA) and the various forms of the generalized gradient approximation (GGA), ${ }^{5-9}$ are widely used and remarkably successful. However, they are not consistently able to attain the very high accuracy of $\sim 0.1 \mathrm{eV}$ required to study many interesting chemical reactions. This is why the provision of better approximations for $E_{\mathrm{xc}}$ is the focus of a substantial research effort.

Much of the recent work on exchange-correlation energy functionals has treated (at least part of) the exchange contribution exactly. As in the optimized effective potential method, ${ }^{10-15}$ an orbital-dependent exchange functional is defined by substituting the one-electron DFT wave functions into the Hartree-Fock expression ${ }^{16}$ for the exchange energy. The correlation energy is then often treated in a semilocal approximation. The successes of the resulting hybrid functionals $\mathrm{s}^{10,17,18}$ in quantum chemistry have reawakened interest in the treatment of exact exchange in solids.

The majority of Hartree-Fock and hybrid DFT calculations of solids ${ }^{19-23}$ have leveraged decades of quantum-

\footnotetext{
${ }^{a)}$ Electronic mail: a.sorouri@imperial.ac.uk
}

chemical experience by using a Gaussian basis set. A little work has also been done in a linear augmented plane-wave (LAPW) framework. ${ }^{24,25}$ The accurate and efficient evaluation of $E_{x}$ in the plane-wave basis used in most solid-state quantum molecular dynamics codes has been surprisingly little discussed in the literature.

In 1998, Chawla and Voth ${ }^{26}$ described an FFT-based algorithm for one $k$-point plane-wave calculations of exchange energies and their functional derivatives with respect to the orbitals. (Related techniques appear to have been used in optimized effective potential cal culations by Kleinman ${ }^{27}$ and Städele et al. ${ }^{14}$ ) Chawla and Voth's method is undoubtedly efficient, but the $k$-space integral that gives the exchange energy has a divergent integrand and simple integration methods such as their single $k$-point approach do not give accurate results. Here we generalize their work to multiple $k$ points and to the calculation of exchange energy densities. We then combine it with an adaptation of Gygi and Baldereschi's ${ }^{28}$ Brillouin-zone integration scheme to obtain a method that is both efficient and accurate. This may prove useful in solid-state Hartree-Fock, optimized effective potential, and hybrid DFT calculations. Our motivation was an ongoing project in which we plan to generate a large database of accurate density-functional exchange energy densities for use in the development and testing of exchangecorrelation functionals for periodic systems.

To test our approach, we have calculated exchange energies and exchange energy densities for several simple periodic systems. The results confirm that the computational requirements scale almost linearly with the number of plane waves and quadratically with the number of $k$ vectors. Atomic units (in which all electromagnetic equations are written in cgs form, and the fundamental constants $\hbar, e^{2}$, and $m_{e}$ are set to unity) have been used throughout.

\section{METHODOLOGY}

\section{A. The exchange energy}

Consider a crystalline system consisting of $L \times L \times L$ identical unit cells. Periodic boundary conditions are applied, 
restricting the Bloch $k$ vectors of the one-electron wave functions to a grid of $L^{3}$ points within the Brillouin zone. (Everything that follows is easily adapted for use with more sophisticated Brillouin-zone sampling schemes. ${ }^{29,30}$ ) We are primarily interested in the infinite solid obtained in the $L \rightarrow \infty$ limit.

In both Hartree-Fock and density-functional theory, the one-electron orbitals may be found either indirectly, by solving a set of self-consistent one-electron equations, or directly, by minimizing the appropriate energy functional. ${ }^{31}$ Regardless of the method used, it is convenient to represent the one-electron orbitals as linear combinations of basis functions. The use of a plane-wave basis set is straightforward and efficient if the atoms have weak pseudopotentials.

The plane-wave expansion of an orbital $\psi_{\mathbf{k} n}(\mathbf{r})$ takes the form:

$$
\psi_{\mathbf{k} n}(\mathbf{r})=\frac{1}{\sqrt{\Omega^{s}}} \sum_{\mathbf{G}} c_{\mathbf{k} n}(\mathbf{G}) e^{i(\mathbf{k}+\mathbf{G}) \cdot \mathbf{r}},
$$

where $\mathbf{k}$ is one of the $L^{3}$ allowed Bloch wave vectors within the Brillouin zone, $n$ is a band index, $\Omega^{\mathrm{s}}$ is the volume of the $L \times L \times L$ system, and the sum is over the reciprocal-lattice vectors $\mathbf{G}$. The expansion coefficients satisfy

$$
\sum_{\mathbf{G}}\left|c_{\mathbf{k} n}(\mathbf{G})\right|^{2}=1
$$

so that $\psi_{\mathbf{k} n}(\mathbf{r})$ is normalized to unity over the whole system.

To calculate the exchange energy from the orbitals, we adapt the fast Fourier transform (FFT)-based approach of Chawla and Voth. ${ }^{26}$ The exchange energy (including the selfexchange term) is given by the standard expression ${ }^{16}$

$$
\begin{aligned}
E_{x}= & -\frac{1}{2} \sum_{\substack{\mathbf{k} n \sigma \\
\text { occ }}} \sum_{\mathbf{k}^{\prime} n^{\prime} \sigma^{\prime}} \delta_{\sigma, \sigma^{\prime}} \int_{\Omega^{s}} \int_{\Omega^{s}} d^{3} r d^{3} r^{\prime} \\
& \times \frac{\psi_{\mathbf{k} n \sigma}^{*}(\mathbf{r}) \psi_{\mathbf{k} n \sigma^{\prime}}\left(\mathbf{r}^{\prime}\right) \psi_{\mathbf{k}^{\prime} n^{\prime} \sigma^{\prime}}(\mathbf{r}) \psi_{\mathbf{k}^{\prime} n^{\prime} \sigma^{\prime}}^{*}\left(\mathbf{r}^{\prime}\right)}{\left|\mathbf{r}-\mathbf{r}^{\prime}\right|},
\end{aligned}
$$

where $\sigma$ is the $z$ component of the electron spin, all integrals are over the whole of the system, and the orbitals are normalized as above. Assuming for simplicity that the system is not spin-polarized, so that $\psi_{n \mathbf{k} \sigma}(\mathbf{r})$ is independent of $\sigma$ and every occupied orbital is occupied by both a spin-up and a spin-down electron, we obtain

$$
\begin{aligned}
E_{x}= & -\frac{1}{4} \sum_{\mathbf{k}_{1} n_{1}} \sum_{\mathbf{k}_{2} n_{2}} f_{\mathbf{k}_{1} n_{1}} f_{\mathbf{k}_{2} n_{2}} \int_{\Omega^{s}} \int_{\Omega^{s}} d^{3} r d^{3} r^{\prime} \\
& \times \frac{e^{-\mu\left|\mathbf{r}-\mathbf{r}^{\prime}\right|} \psi_{\mathbf{k}_{1} n_{1}}^{*}(\mathbf{r}) \psi_{\mathbf{k}_{1} n_{1}}\left(\mathbf{r}^{\prime}\right) \psi_{\mathbf{k}_{2} n_{2}}(\mathbf{r}) \psi_{\mathbf{k}_{2} n_{2}}^{*}\left(\mathbf{r}^{\prime}\right)}{\left|\mathbf{r}-\mathbf{r}^{\prime}\right|},
\end{aligned}
$$

where $e^{-\mu\left|\mathbf{r}-\mathbf{r}^{\prime}\right|}$ is a convergence factor included to ensure that the integrals are well defined and $f_{\mathbf{k} n}$ is the occupation number of state $\psi_{\mathbf{k} n}(\mathbf{r})$. At $T=0$ in a nonmagnetic system, $f_{\mathbf{k} n}$ is 2 for occupied states and 0 for unoccupied states.

Replacing the orbitals by their plane-wave expansions and evaluating the integrals yield (after some algebra) the following expression:

$$
\begin{aligned}
E_{x}= & -\frac{\pi}{\Omega^{s}} \sum_{\mathbf{k}_{1} n_{1}} \sum_{\mathbf{k}_{2} n_{2}} f_{\mathbf{k}_{1} n_{1}} f_{\mathbf{k}_{2} n_{2}} \sum_{\mathbf{G}_{1}, \mathbf{G}^{\prime}, \mathbf{G}_{1}^{\prime}} \\
& \times \frac{c_{\mathbf{k}_{1} n_{1}}^{*}\left(\mathbf{G}_{1}\right) c_{\mathbf{k}_{2} n_{2}}\left(\mathbf{G}_{1}+\mathbf{G}^{\prime}\right) c_{\mathbf{k}_{1} n_{1}}\left(\mathbf{G}_{1}^{\prime}\right) c_{\mathbf{k}_{2} n_{2}}^{*}\left(\mathbf{G}_{1}^{\prime}+\mathbf{G}^{\prime}\right)}{\mu^{2}+\left|\mathbf{k}_{1}-\mathbf{k}_{2}-\mathbf{G}^{\prime}\right|^{2}} .
\end{aligned}
$$

In the limit as $L \rightarrow \infty$, the divergence as $\mathbf{k}_{1} \rightarrow \mathbf{k}_{2}+\mathbf{G}^{\prime}$ becomes integrable and $\mu$ can be set to zero. Assuming this to be understood, we omit $\mu$ from now on.

If we now introduce the correlation function

$$
C_{\mathbf{k}_{1} n_{1}, \mathbf{k}_{2} n_{2}}\left(\mathbf{G}^{\prime}\right)=\sum_{\mathbf{G}} c_{\mathbf{k}_{1} n_{1}}^{*}(\mathbf{G}) c_{\mathbf{k}_{2} n_{2}}\left(\mathbf{G}+\mathbf{G}^{\prime}\right),
$$

we can rewrite $E_{x}$ in the form:

$$
E_{x}=-\frac{\pi}{\Omega^{s}} \sum_{\mathbf{k}_{1} n_{1}} \sum_{\mathbf{k}_{2} n_{2}} f_{\mathbf{k}_{1} n_{1}} f_{\mathbf{k}_{2} n_{2}} \sum_{\mathbf{G}^{\prime}} \frac{\left|C_{\mathbf{k}_{1} n_{1}, \mathbf{k}_{2} n_{2}}\left(\mathbf{G}^{\prime}\right)\right|^{2}}{\left|\mathbf{k}_{1}-\mathbf{k}_{2}-\mathbf{G}^{\prime}\right|^{2}} .
$$

This is the equivalent of Eq. (8) of Ref. 26, generalized to the case where the system to which the periodic boundary conditions are applied consists of many identical small unit cells instead of a large one. By using the extra translational symmetry, we have been able to replace the sum over $\mathbf{g}_{i}$ vectors from Chawla and Voth's expression (which would be equivalent to a sum over $\mathbf{k}$ and $\mathbf{G}$ vectors in our notation) by a sum over $\mathbf{G}$ vectors only. This is advantageous because the $\mathbf{G}$ vectors are on a much coarser grid than the $\mathbf{k}$ vectors, and hence the sum over $\mathbf{G}$ vectors has fewer terms.

\section{B. The exchange energy density}

The exchange energy density $e_{x}(\mathbf{r})$ is not uniquely defined by the equation

$$
E_{x}=\int_{\Omega^{\mathrm{s}}} d^{3} r e_{x}(\mathbf{r})
$$

because any function that integrates to zero can be added to $e_{x}(\mathbf{r})$ without changing $E_{x}$. A unique and, we contend, natural definition of $e_{x}(\mathbf{r})$ may, however, be obtained by omitting one (either) of the integrals over the system in Eq. (4). This yields the following expression:

$$
\begin{aligned}
e_{x}(\mathbf{r})= & -\frac{\pi}{\left(\Omega^{s}\right)^{2}} \sum_{\mathbf{k}_{1} n_{1}} \sum_{\mathbf{k}_{2} n_{2}} f_{\mathbf{k}_{1} n_{1}} f_{\mathbf{k}_{2} n_{2}} \\
& \times \sum_{\mathbf{G}_{1}, \mathbf{G}_{2}} \sum_{\mathbf{G}_{1}^{\prime}, \mathbf{G}_{2}^{\prime}} c_{\mathbf{k}_{1} n_{1}}^{*}\left(\mathbf{G}_{1}\right) c_{\mathbf{k}_{2} n_{2}}\left(\mathbf{G}_{2}\right) \\
& \times \frac{c_{\mathbf{k}_{1} n_{1}}\left(\mathbf{G}_{1}^{\prime}\right) c_{\mathbf{k}_{2} n_{2}}^{*}\left(\mathbf{G}_{2}^{\prime}\right) e^{i\left(\mathbf{G}_{2}-\mathbf{G}_{2}^{\prime}-\mathbf{G}_{1}+\mathbf{G}_{1}^{\prime}\right) \cdot \mathbf{r}}}{\left|\mathbf{k}_{1}+\mathbf{G}_{1}^{\prime}-\mathbf{k}_{2}-\mathbf{G}_{2}^{\prime}\right|^{2}} .
\end{aligned}
$$

If we now expand $e_{x}(\mathbf{r})$ in a Fourier series,

$$
e_{x}(\mathbf{r})=\sum_{\mathbf{G}} e_{x}(\mathbf{G}) e^{i \mathbf{G} \cdot \mathbf{r}},
$$

introduce the vector $\mathbf{G}^{\prime}=\mathbf{G}_{2}^{\prime}-\mathbf{G}_{1}^{\prime}$, and note that the plane waves are linearly independent, we find that 




FIG. 1. The time required to calculate the exchange energy density as a function of the number of plane waves, relative to the time for a 57 planewave calculation. All results are for a system with $L=5$ (i.e., a $5 \times 5 \times 5 k$-point mesh). As expected, the graph is close to linear.

$$
\begin{aligned}
e_{x}(\mathbf{G})= & -\frac{\pi}{\left(\Omega^{s}\right)^{2}} \sum_{\mathbf{k}_{1} n_{1}} \sum_{\mathbf{k}_{2} n_{2}} f_{\mathbf{k}_{1} n_{1}} f_{\mathbf{k}_{2} n_{2}} \\
& \times \sum_{\mathbf{G}^{\prime}} \frac{C_{\mathbf{k}_{1} n_{1}, \mathbf{k}_{2} n_{2}}^{*}\left(\mathbf{G}^{\prime}\right) C_{\mathbf{k}_{1} n_{1}, \mathbf{k}_{2} n_{2}}\left(\mathbf{G}^{\prime}+\mathbf{G}\right)}{\left|\mathbf{k}_{1}-\mathbf{k}_{2}-\mathbf{G}^{\prime}\right|^{2}} .
\end{aligned}
$$

A comparison of Eqs. (7) and (11) confirms that $E_{x}=\Omega^{\mathrm{s}} e_{x}(\mathbf{G}=\mathbf{0})$ as expected.

\section{Efficiency}

For any given choice of $\mathbf{k}_{1}, n_{1}, \mathbf{k}_{2}$, and $n_{2}$, the use of FFTs makes it possible to calculate all $N_{\mathrm{PW}}$ elements of the correlation function $C_{\mathbf{k}_{1} n_{1}, \mathbf{k}_{2} n_{2}}(\mathbf{G})$ in order $N_{\mathrm{PW}} \log N_{\mathrm{PW}}$ operations, where $N_{\mathrm{PW}}$ is the number of plane waves in the basis set. ${ }^{32}$ The $N_{\mathrm{PW}}$ elements of

$$
D_{\mathbf{k}_{1} n_{1}, \mathbf{k}_{2} n_{2}}(\mathbf{G}) \equiv \frac{C_{\mathbf{k}_{1} n_{1}, \mathbf{k}_{2} n_{2}}(\mathbf{G})}{\left|\mathbf{k}_{1}-\mathbf{k}_{2}-\mathbf{G}\right|^{2}}
$$

are then easily obtained. The sum over $\mathbf{G}^{\prime}$ in Eq. (11) is the correlation of $D_{\mathbf{k}_{1} n_{1}, \mathbf{k}_{2} n_{2}}$ and $C_{\mathbf{k}_{1} n_{1}, \mathbf{k}_{2} n_{2}}$, which requires a further $N_{\mathrm{PW}} \log N_{\mathrm{PW}}$ operations. Since the calculation of all $N_{\mathrm{PW}}$ elements of $e_{x}(\mathbf{G})$ requires the evaluation of $N_{\text {occ }}^{2}$ such correlations, where $N_{\text {occ }}$ is the number of occupied states (the number of $k$ points multiplied by the average number of occupied bands per $k$ point), the total computational effort scales as $N_{\mathrm{occ}}^{2} N_{\mathrm{PW}} \log N_{\mathrm{PW}}$. The evaluation of the exchange energy density is not substantially harder than the evaluation of the exchange energy $\Omega^{s} e_{x}(\mathbf{G}=\mathbf{0})$.

The nearly linear scaling with $N_{\mathrm{PW}}$ is optimal, but the quadratic scaling with $N_{\text {occ }}$ (the total number of electrons in the $L \times L \times L$ system) is worse than the linear scaling of an LDA calculation. This emphasizes the importance of using the smallest possible value of $L$ (and hence the smallest possible number of $k$ points) consistent with the required accuracy.

\section{Exchange energy gradients}

The nonlocal exchange operator $\hat{V}_{x}$ acts on an arbitrary state $\phi(\mathbf{r})$ as follows:

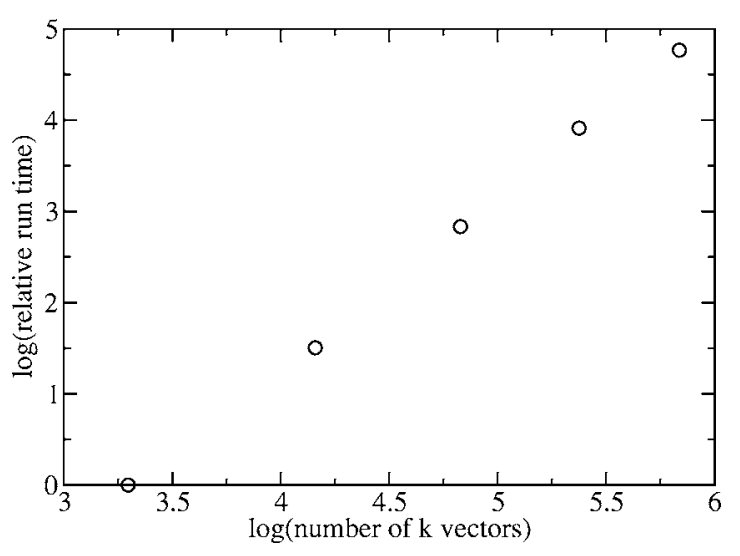

FIG. 2. The logarithm of the time required to calculate the exchange energy density, relative to the time for the smallest system studied, as a function of the logarithm of the number of $k$ vectors, for five systems with $N_{\mathrm{PW}}=515$.

$$
\begin{aligned}
\hat{V}_{x} \phi(\mathbf{r}) & =\int_{\Omega^{s}} d^{3} r^{\prime} V_{x}\left(\mathbf{r}, \mathbf{r}^{\prime}\right) \phi\left(\mathbf{r}^{\prime}\right) \\
& =-\frac{1}{2} \sum_{\mathbf{k} n} f_{\mathbf{k} n} \psi_{\mathbf{k} n}(\mathbf{r}) \int_{\Omega^{s}} d^{3} r^{\prime} \frac{\psi_{\mathbf{k} n}^{*}\left(\mathbf{r}^{\prime}\right) \phi\left(\mathbf{r}^{\prime}\right)}{\left|\mathbf{r}-\mathbf{r}^{\prime}\right|}
\end{aligned}
$$

For a calculation with $N_{k}$ points on the reciprocal/real-space FFT grids, the matrix $V_{x}\left(\mathbf{r}, \mathbf{r}^{\prime}\right)$ has of order of $N_{k} N_{\mathrm{PW}}^{2}$ independent elements ( $\mathbf{r}$ could be any of the $N_{\mathrm{PW}}$ real-space FFT grid points in the unit cell, and $\mathbf{r}^{\prime}$ could be any of the $N_{\mathrm{PW}} N_{k}$ real-space FFT grid points in the system). In insulators, the one-electron density matrix $\rho_{1}\left(\mathbf{r}, \mathbf{r}^{\prime}\right)=\Sigma_{\mathbf{k} n} f_{\mathbf{k} n} \psi_{\mathbf{k} n}(\mathbf{r}) \psi_{\mathbf{k} n}^{*}\left(\mathbf{r}^{\prime}\right)$ dies off rapidly as $\left|\mathbf{r}-\mathbf{r}^{\prime}\right|$ increases and so some of these matrix elements may be small, but it is clearly impossible to evaluate the whole of $V_{x}\left(\mathbf{r}, \mathbf{r}^{\prime}\right)$ quickly.

Fortunately, if the orbitals are obtained via a direct minimization of the energy functional, the full exchange operator is never required. One needs only gradients of the exchange energy: the $N_{\text {occ }}$ functional derivatives $\delta E_{\mathrm{X}} / \delta \psi_{\mathbf{k} n}^{*}(\mathbf{r})$ or the $N_{\text {occ }} N_{\text {PW }}$ partial derivatives $\partial E_{\mathrm{x}} / \partial c_{\mathbf{k} n}^{*}(\mathbf{G})$. These quantities are also required in calculations using the optimized effective potential method. ${ }^{14}$ The two sets of gradients are related via a Fourier transformation,

$$
\frac{\delta E_{x}}{\delta \psi_{\mathbf{k} n}^{*}(\mathbf{r})}=\frac{e^{i \mathbf{k} \cdot \mathbf{r}}}{\sqrt{\Omega^{s}}} \sum \frac{\partial E_{x}}{\partial c_{\mathbf{k} n}^{*}(\mathbf{G})} e^{i \mathbf{G} \cdot \mathbf{r}}
$$

and so the conversion from one set to the other takes of order of $N_{\mathrm{PW}} \log N_{\mathrm{PW}}$ operations per occupied state, or $N_{\text {occ }} N_{\text {PW }} \log N_{\text {PW }}$ operations altogether.

Some straightforward but tedious algebra starting from Eq. (5) shows that

$$
\begin{aligned}
\frac{\partial E_{\mathbf{x}}}{\partial c_{\mathbf{k} n}^{*}(\mathbf{G})}= & -\frac{2 \pi}{\Omega^{s}} f_{\mathbf{k} n} \sum_{\mathbf{k}^{\prime} n^{\prime}} f_{\mathbf{k}^{\prime} n^{\prime}} \\
& \times \sum_{\mathbf{G}^{\prime}} \frac{C_{\mathbf{k} n, \mathbf{k}^{\prime} n^{\prime}}^{*}\left(\mathbf{G}^{\prime}\right) c_{\mathbf{k}^{\prime} n^{\prime}}\left(\mathbf{G}^{\prime}+\mathbf{G}\right)}{\left|\mathbf{k}-\mathbf{k}^{\prime}-\mathbf{G}^{\prime}\right|^{2}} .
\end{aligned}
$$

The sum over $\mathbf{G}^{\prime}$ is yet another correlation function, this time involving $D_{\mathbf{k} n, \mathbf{k}^{\prime} n^{\prime}}$ and $c_{\mathbf{k}^{\prime} n^{\prime}}$. The evaluation of all 


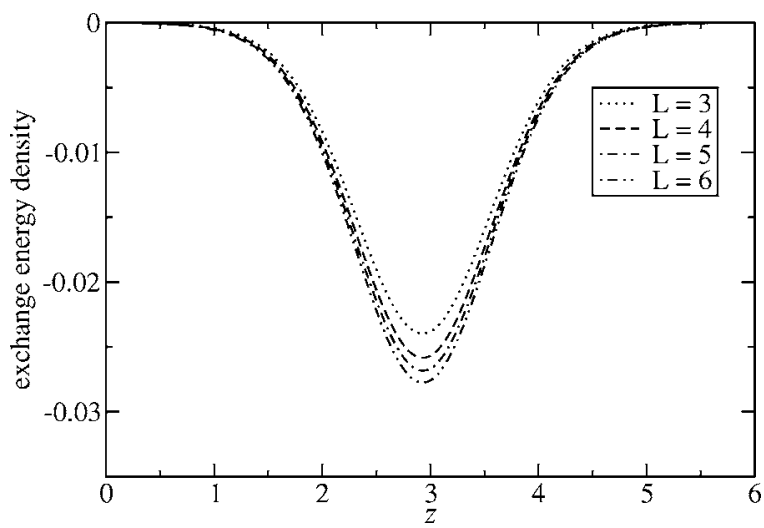

FIG. 3. The exchange energy density per unit cell for a model cosine-wave system, calculated using Eq. (11) for four different values of $L$ (and hence different numbers of $k$ points) and 515 terms in the plane-wave expansion.

$N_{\text {occ }} N_{\text {PW }}$ gradients of $E_{x}$ may therefore be accomplished in order $N_{\text {occ }}^{2} N_{\text {PW }} \log N_{\text {PW }}$ operations.

\section{E. Accuracy}

The above method makes efficient use of FFTs and the correlation theorem but does not yield accurate results. The problem is that the summands in the expressions for $e_{x}(\mathbf{G})$ and $\partial E_{x} / \partial c_{\mathbf{k} n}^{*}(\mathbf{G})$ contain integrable divergences. The summations therefore converge very slowly as the number of $k$ points is increased. (In Chawla and Voth's one $k$-point method, this problem would translate into a very slow convergence with system size.) As shown by Gygi and Baldereschi, ${ }^{28}$ the problem can be cured by adding to and subtracting from the right-hand side of Eq. (11) and (15) a reference term with the same singularities as the exact expression. The application of this technique to Eq. (11) is described below; the application to Eq. (15) is analogous but somewhat simpler.

The first step is to notice that $\psi_{\mathbf{k} n}(\mathbf{r})$ is a periodic function of the Bloch wave vector $\mathbf{k}$ :

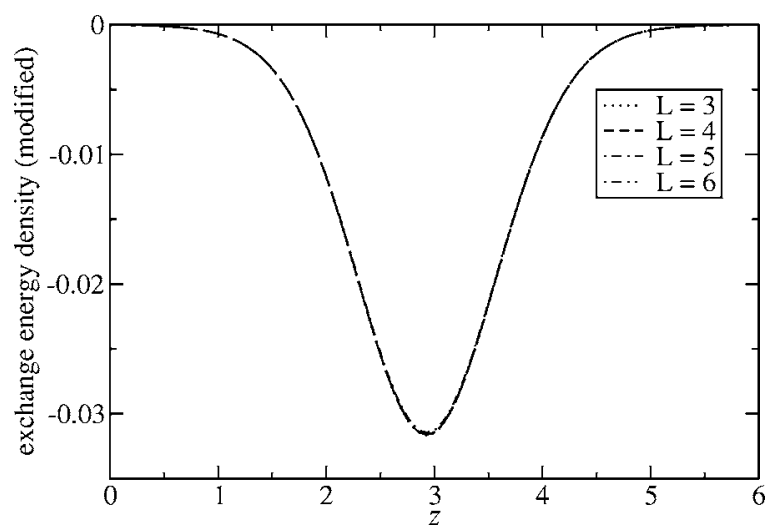

FIG. 4. The exchange energy density for the same system and parameters as in Fig. 3, calculated using the modified expression, Eq. (18).

$$
\psi_{\mathbf{k} n}(\mathbf{r})=\psi_{(\mathbf{k}-\mathbf{G}) n}(\mathbf{r}) .
$$

From Eqs. (1) and (16), it follows that $c_{\left(\mathbf{k}-\mathbf{G}^{\prime}\right) n}\left(\mathbf{G}+\mathbf{G}^{\prime}\right)$ $=c_{\mathbf{k} n}(\mathbf{G})$. Hence, using Eq. (6), we obtain

$$
C_{\mathbf{k}_{1} n_{1},\left(\mathbf{k}_{2}-\mathbf{G}^{\prime}\right) n_{2}}\left(\mathbf{G}+\mathbf{G}^{\prime}\right)=C_{\mathbf{k}_{1} n_{1}, \mathbf{k}_{2} n_{2}}(\mathbf{G}) .
$$

Since the occupation numbers $f_{\mathbf{k} n}$ are also periodic in the Brillouin zone, the coefficient

$$
\begin{aligned}
& -\frac{\pi}{\left(\Omega^{s}\right)^{2}} f_{\mathbf{k}_{1} n_{1}} f_{\left(\mathbf{k}_{1}-\mathbf{G}^{\prime}\right) n_{2}} C_{\mathbf{k}_{1} n_{1},\left(\mathbf{k}_{1}-\mathbf{G}^{\prime}\right) n_{2}}^{*}\left(\mathbf{G}^{\prime}\right) \\
& \quad \times C_{\mathbf{k}_{1} n_{1},\left(\mathbf{k}_{1}-\mathbf{G}^{\prime}\right) n_{2}}\left(\mathbf{G}^{\prime}+\mathbf{G}\right)
\end{aligned}
$$

of the divergent $\mathbf{k}_{2}=\mathbf{k}_{1}-\mathbf{G}^{\prime}$ term in Eq. (11) may be written as

$$
-\frac{\pi}{\left(\Omega^{s}\right)^{2}} f_{\mathbf{k}_{1} n_{1}} f_{\mathbf{k}_{1} n_{2}} C_{\mathbf{k}_{1} n_{1}, \mathbf{k}_{1} n_{2}}^{*}(\mathbf{0}) C_{\mathbf{k}_{1} n_{1}, \mathbf{k}_{1} n_{2}}(\mathbf{G}),
$$

which is independent of $\mathbf{G}^{\prime}$. This shows that the diverging term is periodic and diverges in exactly the same manner at every reciprocal lattice vector.

The next step is to rewrite Eq. (11) as follows:

$$
\begin{aligned}
e_{x}^{\bmod }(\mathbf{G})= & -\frac{\pi}{\left(\Omega^{s}\right)^{2}} \sum_{\mathbf{k}_{1} n_{1}} \sum_{\mathbf{k}_{2} n_{2}}\left[f_{\mathbf{k}_{1} n_{1}} f_{\mathbf{k}_{2} n_{2}} \sum_{\mathbf{G}^{\prime}} \frac{C_{\mathbf{k}_{1} n_{1}, \mathbf{k}_{2} n_{2}}^{*}\left(\mathbf{G}^{\prime}\right) C_{\mathbf{k}_{1} n_{1}, \mathbf{k}_{2} n_{2}}\left(\mathbf{G}^{\prime}+\mathbf{G}\right)}{\left|\mathbf{k}_{1}-\mathbf{k}_{2}-\mathbf{G}^{\prime}\right|^{2}}-f_{\mathbf{k}_{1} n_{1}} f_{\mathbf{k}_{1} n_{2}} C_{\mathbf{k}_{1} n_{1}, \mathbf{k}_{1} n_{2}}^{*}(\mathbf{0}) C_{\mathbf{k}_{1} n_{1}, \mathbf{k}_{1} n_{2}}(\mathbf{G}) F\left(\mathbf{k}_{1}-\mathbf{k}_{2}\right)\right] \\
& -\frac{\pi}{\left(\Omega^{s}\right)^{2}} \sum_{\mathbf{k}_{1} n_{1}} \sum_{n_{2}} f_{\mathbf{k}_{1} n_{1}} f_{\mathbf{k}_{1} n_{2}} C_{\mathbf{k}_{1} n_{1}, \mathbf{k}_{1} n_{2}}^{*}(\mathbf{0}) C_{\mathbf{k}_{1} n_{1}, \mathbf{k}_{1} n_{2}}(\mathbf{G})\left(\sum_{\mathbf{k}_{2}} F\left(\mathbf{k}_{1}-\mathbf{k}_{2}\right)\right)
\end{aligned}
$$

where $F(\mathbf{k})$ is an auxiliary periodic function that diverges like $1 / k^{2}$ as $k$ vanishes. The summand in square brackets is now smooth and may be approximated accurately using a finite quadrature (i.e., a small value of $L$ ). The divergence in the summation over $\mathbf{k}_{2}$ on the third line is handled exactly by noting that the $L \rightarrow \infty$ limit of the summation tends to an integral over the Brillouin zone:

$$
\frac{\Omega^{s}}{(2 \pi)^{3}} \int_{\mathrm{BZ}} F\left(\mathbf{k}_{1}-\mathbf{k}_{2}\right) d \mathbf{k}_{2} .
$$

If the auxiliary function is chosen sensibly, this integral [which must be independent of $\mathbf{k}_{1}$ because $F(\mathbf{k})$ is periodic] can be evaluated exactly.

Gygi and Baldereschi ${ }^{28}$ used a specific $F(\mathbf{k})$ for face- 


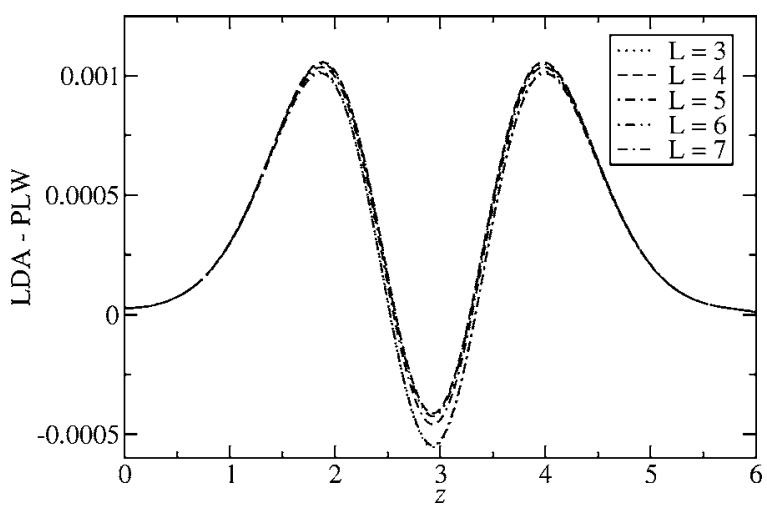

FIG. 5. The function $e_{x}^{\mathrm{LDA}}(z)-e_{x}^{\mathrm{PLW}}(z)$ as a function of $z$ for systems with five different values of $L$.

centred-cubic lattices only. Their choice was efficient but not easy to generalize to arbitrary lattices. We use the simpler and more general (but possibly less efficient) form

$$
F(\mathbf{k})=\sum_{\mathbf{G}} \frac{e^{-\alpha|\mathbf{k}-\mathbf{G}|^{2}}}{|\mathbf{k}-\mathbf{G}|^{2}}
$$

where $\alpha$ is a constant of order 1 that is adjusted to optimize the rate of convergence. The values of $F(\mathbf{k})$ required for the finite quadrature are obtained by precomputing the quickly convergent summation over $\mathbf{G}$ vectors at each quadrature point. The integral of $F(\mathbf{k})$ over the Brillouin zone is equal to $2 \pi \sqrt{\pi / \alpha}$.

\section{RESULTS}

As mentioned above, the use of FFTs and the correlation theorem allows the exchange energy, gradients of the exchange energy, and the exchange energy density to be evaluated efficiently. Figures 1 and 2 confirm that the time taken to calculate the exchange energy density is roughly proportional to the number of plane waves and to the square of the number of $k$ vectors, respectively.

Figure 3 shows the slow convergence with $L$ (or, equivalently, with the number of $k$ points) of the multi- $k$-point generalization of the method of Chawla and Voth, Eq. (11). The calculations were carried out for a model system in which the self-consistent Kohn-Sham potential was defined to be a simple cosine in the $z$ direction, but the slow convergence is a general feature. Figure 4, which shows the corresponding results obtained using the modified expression for the exchange energy density, Eq. (18) demonstrates that the convergence problem has been resolved.

Figure 5 shows a more interesting aspect of the behavior of the same model system. Since the self-consistent KohnSham potential of this system is defined to be a simple cosine wave, the exact Kohn-Sham orbitals and electron density are easily obtained by solving the Kohn-Sham equations nonself-consistently. (The external potential, by contrast, is unknown because we do not know the exact exchangecorrelation functional.) Figure 5 shows the difference between $e_{x}^{\mathrm{LDA}}(z)$, the exchange energy density calculated by substituting the exact electron density into the LDA for exchange, and $e_{\mathrm{x}}^{\mathrm{PLW}}(z)$, the (density-functional) exchange energy density calculated using the plane-wave approach of Eq. (18).

The highest energy level of the cosine-wave system is often degenerate and only partially occupied (depending on the value of $L$ ). In such cases, in order to reduce the finitesize effects (influence of the finite value of $L$ ), Eq. (18) was generalized to noninteger occupations and all degenerate states were occupied equally. It is clear that the remaining finite-size errors are small and that the results are accurate enough to resolve the very small errors in the LDA for exchange. It is interesting that these errors are qualitatively very similar to the errors in the LDA for the full exchangecorrelation energy density studied by Nekovee et al. ${ }^{33,34}$ using quantum Monte Carlo methods.

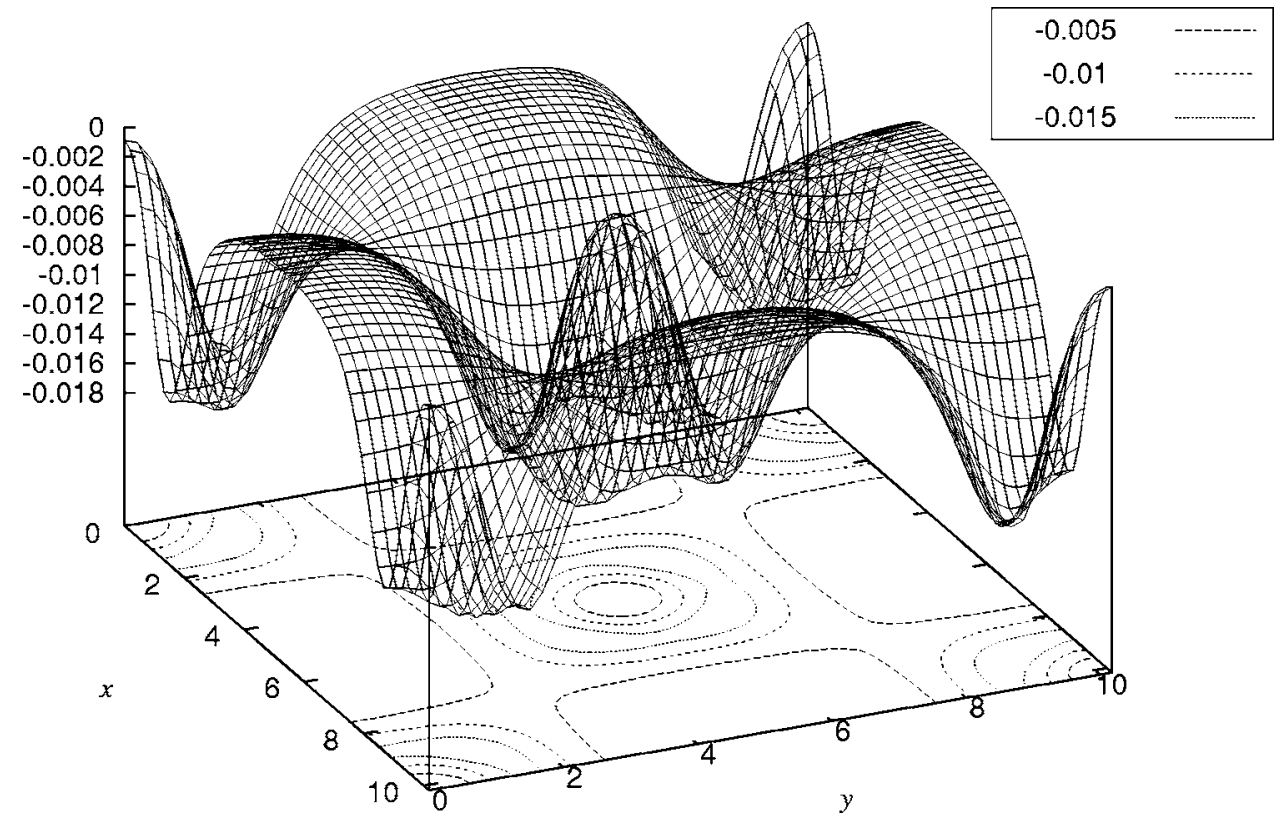

FIG. 6. The LDA exchange energy density $e_{x}^{\mathrm{LDA}}(x, y, z=0)$ for a simple cubic unit cell for silicon. 


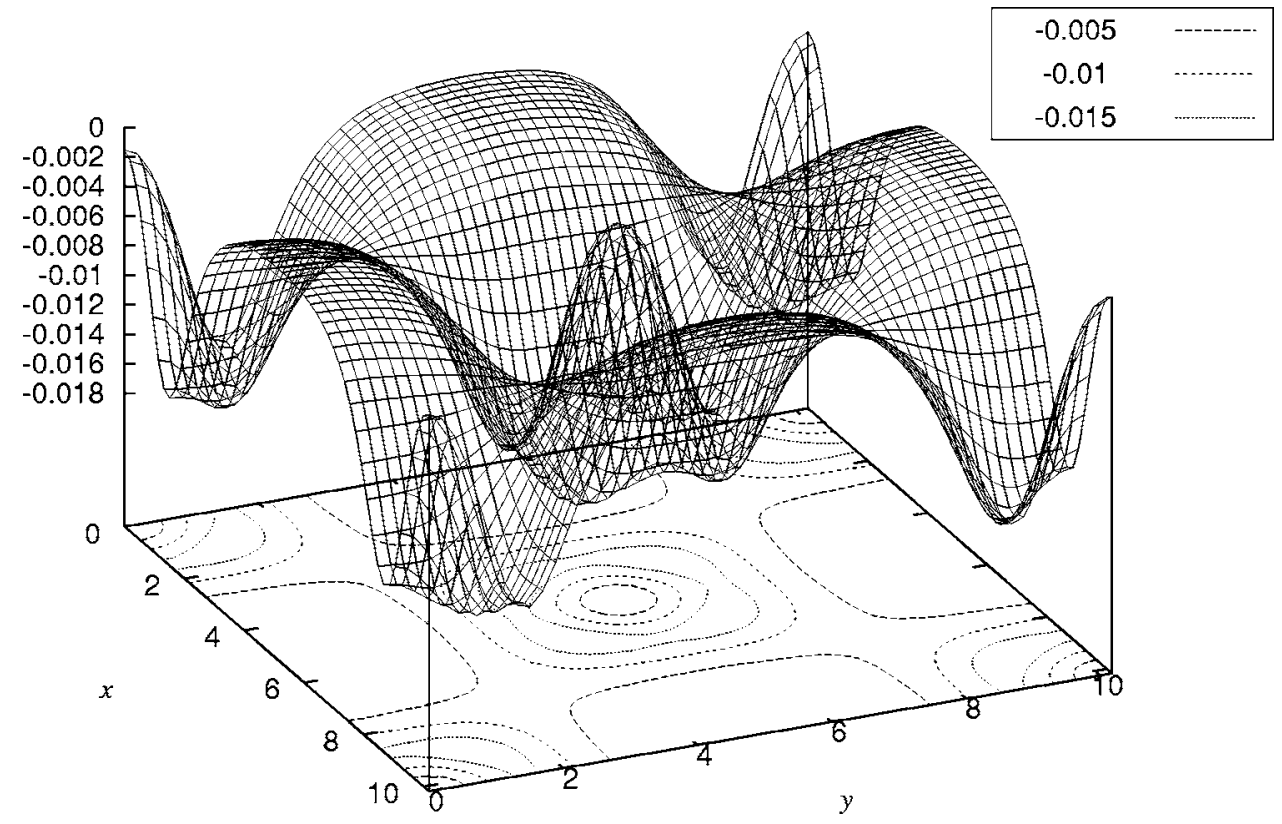

FIG. 7. The exchange energy density (plane-wave approach) $e_{x}^{\mathrm{PLW}}(x, y, z$ $=0$ ) for the same system as Fig. 6 .

As a real example we applied our method to calculate the exchange energy density for silicon, using a TroullierMartins silicon pseudopotential ${ }^{35}$ with four valence electrons. In this case the potential to be solved consists of the local part of the Kohn-Sham potential (which comprises the Hartree potential, the LDA exchange-correlation potential, and the local part of the pseudopotential) and a nonlocal part consisting of the nonlocal part of the pseudopotential. The Hartree and exchange-correlation potentials are produced by a self-consistent DFT calculation using ABINIT, ${ }^{36,37}$ which also provides the local contribution to the pseudopotential. The nonlocal contribution is evaluated by calculating the nonlocal form factors, using the same method as ABINIT (well explained by Nelson et l. $^{38}$ ) to ensure comparable results. Figures 6 and 7 show the exchange energy density in the $x-y$ plane at $z=0$ of a simple cubic unit cell containing four fcc primitive cells and eight silicon atoms, obtained us- ing the LDA and the plane-wave approach of Eq. (18), respectively. Although they appear similar, the errors in the LDA for exchange, shown in Fig. 8, are comparable in magnitude to the errors seen in Fig. 5.

\section{CONCLUSION}

By combining and extending the work of Chawla and Voth $^{26}$ and Gygi and Baldereschi, ${ }^{28}$ we have developed an efficient and highly accurate method for calculating exchange energies, exchange energy densities, and gradients of the exchange energy with respect to wave-function parameters in plane-wave simulations of periodic systems. The computational effort for all these quantities scales like $N_{\text {occ }}^{2} N_{\mathrm{PW}} \log N_{\mathrm{PW}}$, where $N_{\text {occ }}$ is the number of occupied oneelectron orbitals (the number of $k$ points multiplied by the average number of occupied states per $k$ point) and $N_{\mathrm{PW}}$ is

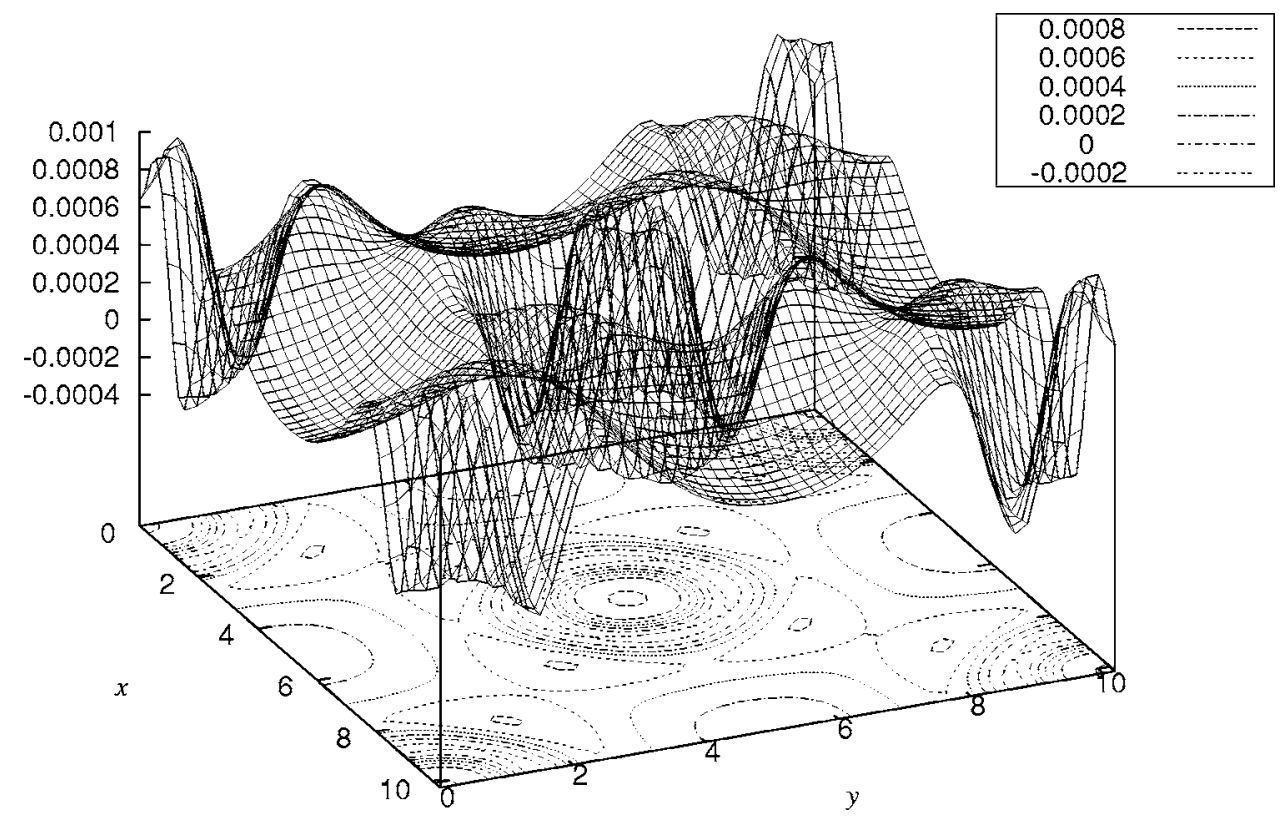

FIG. 8. The difference $e_{x}^{\mathrm{LDA}}(x, y, z$ $=0)-e_{x}^{\mathrm{PLW}}(x, y, z=0)$ between the two methods. 
the number of plane waves in the basis. Accurate results can be obtained using numbers of plane waves and $k$ points comparable to those used in local-density calculations.

The computational effort required to evaluate the gradients of the exchange energy with respect to the wavefunction parameters scales as $N_{\text {occ }}^{2} N_{\text {PW }} \log N_{\text {PW }}$, as opposed to $N_{\text {occ }} N_{\text {PW }} \log N_{\text {PW }}$ in a conventional local-density calculation with a local separable pseudopotential. ${ }^{31}$ The method of Gygi and Baldereschi allows the number of $k$ points and hence $N_{\text {occ }}$ to be kept fairly small, but the extra cost remains substantial. There is also an overhead caused by the need to evaluate correlations of correlations. The FFT-based algorithm used to calculate correlation functions requires a doubling of the number of grid points in each dimension. ${ }^{32}$ Hence, unless one approximates by truncating the FFT grid, it is necessary to pay the computational cost of two successive doublings. A single (untruncated) calculation of the exchange energy density for a system with 87 occupied states and 515 plane waves takes about 6 min. on a $2.4 \mathrm{GHz}$ Pentium personal computer (PC).

Although plane-wave-based quantum molecular dynamics simulations incorporating exact exchange will remain challenging, one-off calculations of exchange energies, their gradients with respect to wave-function parameters, and exchange energy densities are straightforward. Quantities sensitive to the choice of exchange-correlation functional can be investigated using Hartree-Fock theory or hybrid density functionals at reasonable cost and with high accuracy.

\footnotetext{
${ }^{1}$ P. Hohenberg and W. Kohn, Phys. Rev. 136, B864 (1964).

${ }^{2}$ W. Kohn and L. Sham, Phys. Rev. 140, A1133 (1965).

${ }^{3}$ R. M. Dreizler and E. K. U. Gross, Density Functional Theory (Springer, Berlin, 1990).

${ }^{4}$ R. G. Parr and W. Yang, Density Functional Theory of Atoms and Molecules (Oxford University Press, Oxford, 1988).

${ }^{5}$ D. Langreth and M. Mehl, Phys. Rev. B 28, 1809 (1983).

${ }^{6}$ J. P. Perdew and Y. Wang, Phys. Rev. B 33, 8800 (1986).

${ }^{7}$ A. D. Becke, Phys. Rev. A 38, 3098 (1988).

${ }^{8}$ J. P. Perdew, J. A. Chevary, S. H. Vosko, K. A. Jackson, M. R. Pederson, D. J. Singh, and C. Fiolhais, Phys. Rev. B 46, 6671 (1992).
}

${ }^{9}$ J. P. Perdew, K. Burke, and M. Ernzerhof, Phys. Rev. Lett. 77, 3865 (1996).

${ }^{10}$ R. M. Martin, Electronic Structure: Basic Theory and Practical Methods (Cambridge University Press, Cambridge, 2004).

${ }^{11}$ D. M. Bylander and L. Kleinman, Phys. Rev. B 52, 14566 (1995).

${ }^{12}$ M. E. Casida, in Recent Development and Applications of Density Functional Theory, edited by J. M. Seminario (Elsevier, Amsterdam, 1996), p. 391.

${ }^{13}$ T. Grabo and E. K. U. Gross, Int. J. Quantum Chem. 64, 95 (1997).

${ }^{14}$ M. Städele, M. Moukara, J. A. Majewski, and P. Vogl, Phys. Rev. B 59, 10031 (1999).

${ }^{15}$ S. Kummel and J. P. Perdew, Phys. Rev. B 68, 035103 (2003).

${ }^{16}$ A. Szabo and N. S. Ostlund, Modern Quantum Chemistry: Introduction of Advanced Electronic Structure Theory (Dover, Mineola, NY, 1996), unabridged reprinting of the 1989 edition.

${ }^{17}$ A. D. Becke, J. Chem. Phys. 98, 5648 (1993).

${ }^{18}$ M. Ernzerhof and G. E. Scuseria, J. Chem. Phys. 110, 5029 (1999).

${ }^{19}$ A. Wander and N. M. Harrison, Surf. Sci. Lett. 457, L342 (2000).

${ }^{20}$ T. Bredow and A. R. Gerson, Phys. Rev. B 61, 5194 (2000).

${ }^{21}$ J. Muscat, A. Wander, and N. M. Harrison, Chem. Phys. Lett. 342, 397 (2001).

${ }^{22}$ N. C. Wilson, J. Muscat, D. Mkhonto, P. E. Ngoepe, and N. M. Harrison, Phys. Rev. B 71, 075202 (2005).

${ }^{23}$ E. Orhan, J. A. Varela, A. Zenatti, M. F. C. Gurgel, F. M. Pontes, E. R. Leite, E. Longo, P. S. Pizani, A. Beltrán, and J. Andrés, Phys. Rev. B 71, 085113 (2005).

${ }^{24}$ S. Massidda, M. Posternak, and A. Baldereschi, Phys. Rev. B 48, 5058 (1993).

${ }^{25}$ A. V. Nikolaev and P. N. Dyachkov, Int. J. Quantum Chem. 89, 57 (2002).

${ }^{26}$ S. Chawla and G. Voth, J. Chem. Phys. 108, 4697 (1998).

${ }^{27}$ L. Kleinman, Phys. Rev. B 49, 14197 (1994).

${ }^{28}$ F. Gygi and A. Baldereschi, Phys. Rev. B 34, 4405 (1986).

${ }^{29}$ A. Baldereschi, Phys. Rev. B 7, 5212 (1972).

${ }^{30}$ H. J. Monkhorst and J. D. Pack, Phys. Rev. B 13, 5188 (1976).

${ }^{31}$ M. C. Payne, M. P. Teter, D. C. Allan, T. C. Arias, and J. D. Joannopoulos, Rev. Mod. Phys. 64, 1045 (1992).

${ }^{32}$ E. O. Brigham, The Fast Fourier Transform and its Applications (Prentice Hall, Englewood Cliffs, NJ, 1988).

${ }^{33}$ M. Nekovee, W. M. C. Foulkes, and R. J. Needs, Phys. Rev. Lett. 87, 036401 (2001).

${ }^{34}$ M. Nekovee, W. M. C. Foulkes, and R. J. Needs, Phys. Rev. B 68, 235108 (2003).

${ }^{35}$ N. Troullier and J. L. Martins, Phys. Rev. B 43, 1993 (1991).

${ }^{36}$ X. Gonze, J. M. Beuken, R. Caracas et al., Comput. Mater. Sci. 25, 478 (2002).

${ }^{37}$ X. Gonze, G. M. Rignanese, M. Verstraete et al., Z. Kristallogr. 220, 558 (2005).

${ }^{38}$ J. S. Nelson, S. J. Plimpton, and M. P. Sears, Phys. Rev. B 47, 1765 (1993). 